Article

\title{
Numerical and Analytical Investigation of an Unsteady Thin Film Nanofluid Flow over an Angular Surface
}

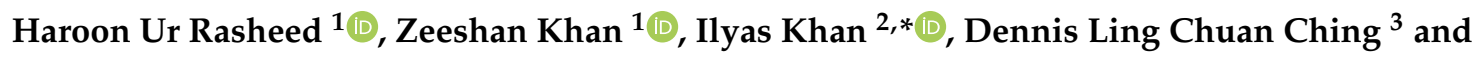 \\ Kottakkaran Sooppy Nisar ${ }^{4}$ (i) \\ 1 Sarhad University of Science and IT, Peshawar, Kpk 25000, Pakistan \\ 2 Faculty of Mathematics and Statistics, Ton Duc Thang University, Ho Chi Minh City 72915, Vietnam \\ 3 Fundamental and Applied Science Department, Universiti Teknologi Petronas, 32610 Perak, Malaysia \\ 4 Department of Mathematics, College of Arts and Science, Prince Sattam bin Abdulaziz University, \\ Wadi Al-Dawaser 11991, Saudi Arabia \\ * Correspondence: ilyaskhan@tdtu.edu.vn
}

Received: 3 May 2019; Accepted: 5 June 2019; Published: 1 August 2019

\begin{abstract}
In the present study, we examine three-dimensional thin film flow over an angular rotating disk plane in the presence of nanoparticles. The governing basic equations are transformed into ordinary differential equations by using similarity variables. The series solution has been obtained by the homotopy asymptotic method (HAM) for axial velocity, radial velocity, darning flow, induced flow, and temperature and concentration profiles. For the sake of accuracy, the results are also clarified numerically with the help of the BVPh2- midpoint method. The effect of embedded parameters such as the Brownian motion parameter $\mathrm{Nb}$, Schmidt number $\mathrm{Sc}$, thermophoretic parameter and Prandtl number $\mathrm{Pr}$ are explored on velocity, temperature and concentration profiles. It is observed that with the increase in the unsteadiness factor $S$, the thickness of the momentum boundary layer increases, while the Sherwood number $S c$, with the association of heat flow from sheet to fluid, reduces with the rise in $S$ and results in a cooling effect. It is also remarkable to note that the thermal boundary layer increases with the increase of the Brownian motion parameter $\mathrm{Nb}$ and Prandtl number $\mathrm{Pr}$, hindering the cooling process resulting from heat transfer.
\end{abstract}

Keywords: numerical and analytical solutions; unsteady flow; nanofluid; thin-film flow; angular surface

\section{Introduction}

The physical interpretation of the thin film has been highlighted by many researchers, engineers and scientists. Miladinova et al. [1] studied the thin film of a power law liquid over an inclined plate. The effects of slip conditions on the thin film flow of third grade fluid has been investigated by Gul et al. [2] for the lifting and drainage problem with constant viscosity. Similarly, Khalid and Vafai [3] investigated hydrodynamic squeezed flow and heat transfer over a sensor surface. Siddique et al. [4] studied thin film flow of non-Newtonian fluid over a moving belt. In another study, the same author [5] investigated the thin film flow of a fourth-grade fluid. Costa and Macedonio [6] showed that an increase in velocity may produce additional growth of local temperature. The variable viscosity effect has been analyzed on the thin film unsteady flow by Nadeem and Awais [7]. MHD flow of third grade fluid with variable viscosity has been investigated by Elahi and Riaz [8]. The approximate analytical solution of the third-grade fluid filled with a porous medium through a parallel plate has been found by Aksoy et al. [9]. 
The majority of the literature deals with the flow of fluid as a base fluid having low thermal conductivity. The outputs of such kinds of thermal systems are very poor. In order to enhance the thermal performance of the base fluid, small size particles, known as nanoparticles are dispersed in the base fluid. Sheikholeslami [10] investigated the nanofluid spray over a rotating and inclined disk. The effect of slip conditions on the peristaltic flow of a Jeffrey fluid with a Newtonian fluid was studied by Vajravelu et al. [11]. Prasad et al. [12] investigated magneto hydrodynamic mixed convicted heat flow over a nonlinear sheet with temperature dependent viscosity. Similarly, Awati [13] carried out an analysis of MHD viscous flow with a heat source. Series and analytical solutions have been obtained and the effect of emerging parameters were discussed through graphs.

The time-dependent fluid flow also has important applications in the field of engineering and applied sciences. Attia [14] examined the fluid flow in the presence of suction and injection over a rotating plane in the presence of nanoparticles. MHD unsteady flow of a nonliquid through a permeable vertically extending medium has been investigated numerically by Freidoonimehr et al. [15]. Makinde et al. [16] extended their effort with the insertion of variable viscosity. Akbarr et al. [17] examined the 2D streaming of a nonliquid using a magnetic field and numerical results were obtained by the shooting method. Ramzann et al. [18] investigated the MHD stream of micropolar nonliquids over a rotating disk with partial slip conditions. It is clear that the physical problems have been frequently modeled using non-linear differential equations. For the solutions of such non-linear problems, several analytical and numerical techniques are used such as the homotopy asymtotic method (HAM) [19], homotopy perturbation method (HPM) [20], optimal homotopy asymtotic method (OHAM) [21], Runge-Kutta fourth order method [22,23] and the finite deference method [24]. Saeed et al. [25] studied the three-dimensional flow of Casson thin-film nanofluids over an angular rotating surface associated with a heat source, and the thermal effect. Binding [26] numerically studied the wire coating process for the polymer melts inelastic constitutive model. Similarly, Nayake et al. [27] used third grade fluid as a wire coating with variable viscosity. Numerical expression has been obtained for the velocity and temperature profiles. Recently Salem et al. [28] and Bhukta et al. [29] also numerically investigated the MHD flow of time dependent viscosity and thermal conductivity, as well as the heat transfer effect on viscoelastic fluids over a stretching sheet.

On behalf of the above important discussion, the prime objective of this study is to analyze the impact of spraying a nanofluid over an inclined rotating plane as a cooling application. The converted differential equations were solved analytically by HAM [30-34]. In the wake of utilizing appropriate similarity variables, the final form of the boundary value problem was clarified numerically with the help of the BVPh2-midpoint method. The physical emerging parameters are portrayed through tables and graphs.

\section{Problem Formulation}

Consider a three-dimensional unsteady nanofluid thin-film flow over an angular disk. The angular velocity with which the disk is rotating in its own plane is denoted by $\Omega$ as shown in Figure 1.

The inclined disk makes an angle $\beta$ with the horizontal axis. The thickness of the nanofluid film is indicated by $h$ and $W$ stands for the spraying velocity. The thickness of the liquid film is very small as compared to the radius of the disk and therefore the end effect is ignored. The gravitational acceleration $\bar{g}$ is acting as usual in the downward direction. $T_{0}$ stands for the temperature at the film surface, while $T_{w}$ stands for the temperature at the disk surface. Similarly, the concentration at the film surface is $C_{0}$ and on the disk surface is $C_{h}$. The ambient pressure $p_{0}$ is kept constant at the surface of the film and as a result, the pressure becomes only a function of $z$. The viscous dissipation is ignored, and the basic governing equations of continuity, momentum boundary layer, thermal boundary layer and mass for the unsteady state are given as

$$
\frac{\partial u}{\partial x}+\frac{\partial v}{\partial y}+\frac{\partial w}{\partial z}=0
$$




$$
\begin{gathered}
\rho_{f}\left(\frac{\partial u}{\partial t}+u \frac{\partial u}{\partial x}+v \frac{\partial u}{\partial y}+w \frac{\partial u}{\partial z}\right)=\mu\left(\frac{\partial^{2} u}{\partial x^{2}}+\frac{\partial^{2} u}{\partial y^{2}}+\frac{\partial^{2} u}{\partial z^{2}}\right)+\rho_{f} \bar{g} \operatorname{Sin} \beta \\
\rho_{f}\left(\frac{\partial v}{\partial t}+u \frac{\partial v}{\partial x}+v \frac{\partial v}{\partial y}+w \frac{\partial v}{\partial z}\right)=\mu\left(\frac{\partial^{2} v}{\partial x^{2}}+\frac{\partial^{2} v}{\partial y^{2}}+\frac{\partial^{2} v}{\partial z^{2}}\right) \\
\rho_{f}\left(\frac{\partial w}{\partial t}+u \frac{\partial w}{\partial x}+v \frac{\partial w}{\partial y}+w \frac{\partial w}{\partial z}\right)=\mu\left(\frac{\partial^{2} w}{\partial x^{2}}+\frac{\partial^{2} w}{\partial y^{2}}+\frac{\partial^{2} w}{\partial z^{2}}\right)+\rho_{f} \bar{g} \operatorname{Cos} \beta-p_{z} \\
\frac{\partial T}{\partial t}+u\left(\frac{\partial T}{\partial x}\right)+v\left(\frac{\partial T}{\partial y}\right)+w\left(\frac{\partial T}{\partial z}\right)=\alpha\left(\frac{\partial^{2} T}{\partial x^{2}}+\frac{\partial^{2} T}{\partial y^{2}}+\frac{\partial^{2} T}{\partial z^{2}}\right) \\
-\frac{\left(\rho c_{p}\right)_{p}}{\left(\rho c_{p}\right)_{f}}\left[D_{B}\left\{\frac{\partial C}{\partial x} \frac{\partial T}{\partial x}+\frac{\partial C}{\partial y} \frac{\partial T}{\partial y}+\frac{\partial C}{\partial z} \frac{\partial T}{\partial z}\right\}+\frac{D_{T}}{T}\left\{\left(\frac{\partial T}{\partial x}\right)^{2}+\left(\frac{\partial T}{\partial y}\right)^{2}+\left(\frac{\partial T}{\partial z}\right)^{2}\right\}\right] \\
\frac{\partial C}{\partial t}+u\left(\frac{\partial C}{\partial x}\right)+v\left(\frac{\partial C}{\partial y}\right)+w\left(\frac{\partial C}{\partial z}\right)=D_{B}\left(\frac{\partial^{2} C}{\partial x^{2}}+\frac{\partial^{2} C}{\partial y^{2}}+\frac{\partial^{2} C}{\partial z^{2}}\right)+\left(\frac{D_{T}}{T_{0}}\right)\left(\frac{\partial^{2} T}{\partial x^{2}}+\frac{\partial^{2} T}{\partial y^{2}}+\frac{\partial^{2} T}{\partial z^{2}}\right) .
\end{gathered}
$$
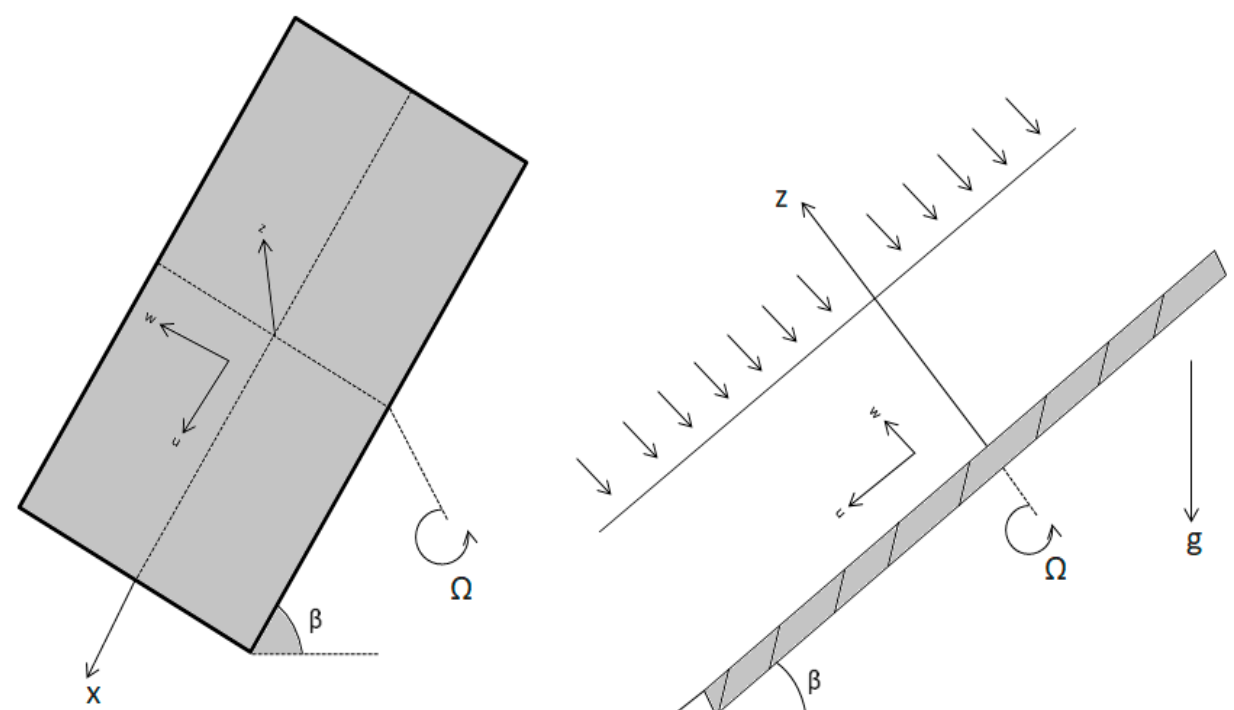

Figure 1. Diagram of the physical problem.

The boundary conditions are defined as

$$
\begin{aligned}
& u=-\Omega y, v=-\Omega x, w=0, T=T_{W}, C=C_{h} \text { at } z=0, \\
& u_{z}=0, v_{z}=0, w=-W, T=T_{0}, C=C_{0}, p=p_{0}, \text { at } z=h .
\end{aligned}
$$

Let us consider the transformations

$$
\begin{aligned}
& u=\frac{-\Omega y}{i-b t} g(\eta)+\frac{\Omega x}{1-b t} f^{\prime}(\eta)+\frac{\bar{g}}{\sqrt{1-b t}} k(\eta) \operatorname{Sin} \frac{\beta}{\Omega^{\prime}}, \\
& v=\frac{-\Omega x}{i-b t} g(\eta)+\frac{\Omega y}{1-b t} f^{\prime}(\eta)+\frac{\frac{\bar{g}}{\sqrt{1-b t}}}{i}(\eta) \operatorname{Sin} \frac{\beta}{\Omega^{\prime}}, \\
& w=-2 \sqrt{\frac{\Omega v_{f}}{1-b t}} f(\eta), \eta \theta(\eta)=\frac{T-T_{w}}{T_{0}-T_{w}}, \\
& \eta \phi(\eta)=\frac{C-C_{w}}{C_{0}-C_{w}}, \eta=z \sqrt{\frac{\Omega}{v_{f}(1-b t)}} .
\end{aligned}
$$

Then, the transformations defined in Equation (8) are inserted into Equations (2)-(7), such that Equation (1) is verified identically and Equations (2)-(6) yield in the following form:

$$
f^{\prime \prime \prime}(\eta)-\left(f^{\prime}(\eta)\right)^{2}+(g(\eta))^{2}-2 f(\eta) f^{\prime \prime}(\eta)-S\left(f^{\prime}(\eta)+\frac{\eta}{2} f^{\prime \prime}(\eta)\right)=0
$$




$$
\begin{gathered}
k^{\prime \prime}(\eta)-k(\eta) f^{\prime}(\eta)-h(\eta) g(\eta)+2 f(\eta) k^{\prime}(\eta)+1-\frac{S}{2}\left(k(\eta)+\eta k^{\prime}(\eta)\right)=0 \\
g^{\prime \prime}(\eta)-2 g(\eta) f^{\prime}(\eta)+2 g^{\prime}(\eta) f(\eta)-S\left(g(\eta)+\frac{\eta}{2} g^{\prime}(\eta)\right)=0 \\
h^{\prime \prime}(\eta)-k(\eta) g(\eta)-h(\eta) f^{\prime}(\eta)+2 f(\eta) h^{\prime}(\eta)-\frac{S}{2}\left(h(\eta)-\eta h^{\prime}(\eta)\right)=0 .
\end{gathered}
$$

If temperature and concentration are a function of the distance $z$ only, Equations (5) and (6) become

$$
\begin{gathered}
\theta^{\prime \prime}(\eta)+2 \operatorname{Pr} f(\eta) \theta^{\prime}(\eta)+N b \phi^{\prime}(\eta) \theta^{\prime}(\eta)+N t\left(\theta^{\prime}(\eta)\right)^{2}+\frac{S}{2}\left(\eta \theta^{\prime}+\eta^{2} \theta^{\prime \prime}\right)=0, \\
\phi^{\prime \prime}(\eta)+2 S c f(\eta) \phi^{\prime}(\eta)+\frac{N t}{N b} \theta^{\prime \prime}(\eta)+\frac{S}{2}\left(\eta \phi^{\prime}+\eta^{2} \phi^{\prime \prime}\right)=0, \\
f(0)=0, f^{\prime}(0)=0, f^{\prime \prime}(\delta)=0, \\
g(0)=0, g^{\prime}(\delta)=0, \\
k(0)=0, k^{\prime}(\delta)=0, \\
h(0)=0, h^{\prime}(\delta)=0, \\
\theta(0)=0, \theta^{\prime}(\delta)=1, \\
\phi(0)=0, \phi^{\prime}(\delta)=1 .
\end{gathered}
$$

Here the Prandtl number $(\mathrm{Pr})$, Schmidt number $(\mathrm{Sc})$, Brownian motion parameter $(\mathrm{Nb})$, and thermophoretic parameter $(N t)$ are defined as

$$
\operatorname{Pr}=\frac{\mu}{\rho_{f} \alpha}, S c=\frac{\mu}{\rho_{f} D^{\prime}}, N b=\frac{(\rho c)_{p} D_{b}\left(C_{h}\right)}{\left\{(\rho c)_{f} \alpha\right\}}, N t=\frac{(\rho c)_{p} D_{T}\left(T_{h}\right)}{\left\{(\rho c)_{f} \alpha T_{c}\right\}}, S=\frac{\alpha}{\Omega},
$$

where $\delta$ is the constant normalized thickness as

$$
\delta=\varepsilon \sqrt{\frac{\Omega}{v_{f}(1-b t)}} .
$$

This is known through the condensation or spraying velocity as

$$
f(\delta)=\frac{w}{2 \sqrt{\Omega v}}=\alpha .
$$

The pressure can be found by integrating Equation (4). For $\operatorname{Pr}=0$, by using $\theta(\delta)=1$, the exact solution is

$$
\theta^{\prime}(0)=\frac{1}{\delta}
$$

Equation (17) stands for an asymptotic limit for a small $\delta$. The decrease of $\theta(0)$ for growing $\delta$ is not monotonic as can be understood from the waviness of the curves for big Pr:

$$
N u=\frac{\left(\frac{\partial T}{\partial z}\right)_{w}}{T_{0}-T_{w}}=\delta \theta^{\prime}(0)
$$

Similarly, the Sherwood number can be defined as

$$
S h=\frac{\left(\frac{\partial T}{\partial z}\right)_{w}}{C_{0}-C_{w}}=\delta \phi^{\prime}(0) .
$$




\section{Solution Methodology}

An optimal homotopy analysis method and BVPh2-midpoint method are implemented in the present analysis for the solution of the non-linear ordinary differential Equations (9)-(14) subject to the boundary conditions given in Equation (15). The set techniques are utilized to get the solutions for highly non-linear equations. The optimal HAM [27-31] gives better results compared with perturbation techniques and other conventional investigative techniques. Firstly, the optimal HAM gives us a remarkable flexibility to pick the equation type of linear sub-problems. Secondly, the optimal HAM works regardless of the possibility that any small or large physical parameters in determining equations and the boundary/initial conditions do not exist. Particularly, unlike perturbation and other analytic techniques, the optimal HAM gives us an advantageous approach to guarantee the convergence of a series solution by presenting the supposed convergence control parameter into the series solution. The comparison of the HAM and numerical solution is given in Tables 1-6 and Figures 2-7, while the graphical representation for the 10th-order approximation displays the error decay in Figure 8.

Table 1. Comparisons of the homotopy asymptotic method (HAM) and numerical solution.

\begin{tabular}{cccc}
\hline$f(\eta)$ & HAM Solution & Numerical Solution & Absolute Error \\
\hline 0.0 & 0.000000 & $-4.113240 \times 10^{-9}$ & $4.113240 \times 10^{-9}$ \\
\hline 0.1 & 0.095967 & 0.095967 & $2.757140 \times 10^{-9}$ \\
\hline 0.2 & 0.184817 & 0.184817 & $2.395770 \times 10^{-9}$ \\
\hline 0.3 & 0.267754 & 0.267754 & $1.427160 \times 10^{-9}$ \\
\hline 0.4 & 0.345761 & 0.345761 & $9.250300 \times 10^{-10}$ \\
\hline 0.5 & 0.419668 & 0.419668 & $6.552510 \times 10^{-10}$ \\
\hline 0.6 & 0.490197 & 0.490197 & $2.840370 \times 10^{-10}$ \\
\hline 0.7 & 0.558005 & 0.558005 & $9.286170 \times 10^{-10}$ \\
\hline 0.8 & 0.623724 & 0.623724 & $1.115280 \times 10^{-9}$ \\
\hline 0.9 & 0.687991 & 0.687991 & $6.833170 \times 10^{-10}$ \\
\hline 1.0 & 0.751489 & 0.751489 & $2.694550 \times 10^{-10}$ \\
\hline
\end{tabular}

Table 2. Comparisons of the HAM and numerical solution.

\begin{tabular}{cccc}
\hline $\boldsymbol{k}(\boldsymbol{\eta})$ & HAM Solution & Numerical Solution & Absolute Error \\
\hline 0.0 & 0.000000 & $8.431700 \times 10^{-11}$ & $8.431700 \times 10^{-11}$ \\
\hline 0.1 & 0.083047 & 0.083047 & $8.194100 \times 10^{-10}$ \\
\hline 0.2 & 0.156141 & 0.156141 & $1.507400 \times 10^{-9}$ \\
\hline 0.3 & 0.218995 & 0.218995 & $2.567920 \times 10^{-9}$ \\
\hline 0.4 & 0.271888 & 0.271888 & $3.456570 \times 10^{-9}$ \\
\hline 0.5 & 0.315142 & 0.315142 & $4.203450 \times 10^{-9}$ \\
\hline 0.6 & 0.349196 & 0.349196 & $4.967530 \times 10^{-9}$ \\
\hline 0.7 & 0.374581 & 0.374581 & $5.555180 \times 10^{-9}$ \\
\hline 0.8 & 0.391897 & 0.391897 & $5.900650 \times 10^{-9}$ \\
\hline 0.9 & 0.401789 & 0.401789 & $6.210250 \times 10^{-9}$ \\
\hline 1.0 & 0.404932 & 0.404932 & $1.059580 \times 10^{-8}$ \\
\hline
\end{tabular}


Table 3. Comparisons of the HAM and numerical solution.

\begin{tabular}{cccc}
\hline$g(\eta)$ & HAM Solution & Numerical Solution & Absolute Error \\
\hline 0.0 & 0.000000 & $-8.866030 \times 10^{-11}$ & $8.866030 \times 10^{-10}$ \\
\hline 0.1 & -0.008248 & -0.008248 & $1.092590 \times 10^{-10}$ \\
\hline 0.2 & -0.016231 & -0.016231 & $6.850590 \times 10^{-10}$ \\
\hline 0.3 & -0.023721 & -0.023721 & $1.199930 \times 10^{-10}$ \\
\hline 0.4 & -0.030534 & -0.030534 & $1.572520 \times 10^{-10}$ \\
\hline 0.5 & -0.036521 & -0.036521 & $1.944400 \times 10^{-10}$ \\
\hline 0.6 & -0.041566 & -0.041566 & $2.672430 \times 10^{-10}$ \\
\hline 0.7 & -0.045583 & -0.045583 & $3.628050 \times 10^{-10}$ \\
\hline 0.8 & -0.048506 & -0.048506 & $3.482500 \times 10^{-10}$ \\
\hline 0.9 & -0.050288 & -0.050288 & $3.068110 \times 10^{-10}$ \\
\hline 1.0 & -0.050890 & -0.050890 & $7.346430 \times 10^{-10}$ \\
\hline
\end{tabular}

Table 4. Comparisons of the HAM and numerical solution.

\begin{tabular}{cccc}
\hline $\boldsymbol{h}(\boldsymbol{\eta})$ & HAM Solution & Numerical Solution & Absolute Error \\
\hline 0.0 & 0.000000 & $-6.298860 \times 10^{-11}$ & $6.298860 \times 10^{-11}$ \\
\hline 0.1 & 0.012496 & 0.012496 & $4.280720 \times 10^{-11}$ \\
\hline 0.2 & 0.024589 & 0.024589 & $4.879770 \times 10^{-11}$ \\
\hline 0.3 & 0.035923 & 0.035923 & $1.257860 \times 10^{-11}$ \\
\hline 0.4 & 0.046200 & 0.046200 & $9.318280 \times 10^{-11}$ \\
\hline 0.5 & 0.055181 & 0.055181 & $2.305180 \times 10^{-10}$ \\
\hline 0.6 & 0.062687 & 0.062687 & $3.234660 \times 10^{-10}$ \\
\hline 0.7 & 0.068598 & 0.068598 & $4.674580 \times 10^{-10}$ \\
\hline 0.8 & 0.072833 & 0.072833 & $6.805310 \times 10^{-10}$ \\
\hline 0.9 & 0.075371 & 0.075371 & $8.340060 \times 10^{-10}$ \\
\hline 1.0 & 0.076213 & 0.076213 & $2.122270 \times 10^{-9}$ \\
\hline
\end{tabular}

Table 5. Comparisons of the HAM and numerical solution.

\begin{tabular}{cccc}
\hline $\boldsymbol{\theta}(\boldsymbol{\eta})$ & HAM Solution & Numerical Solution & Absolute Error \\
\hline 0.0 & 0.000000 & $-8.964480 \times 10^{-9}$ & $8.964480 \times 10^{-9}$ \\
\hline 0.1 & 0.171025 & 0.171025 & $7.561540 \times 10^{-9}$ \\
\hline 0.2 & 0.323916 & 0.323916 & $5.991460 \times 10^{-9}$ \\
\hline 0.3 & 0.458661 & 0.458661 & $1.565540 \times 10^{-9}$ \\
\hline 0.4 & 0.576014 & 0.576014 & $5.012150 \times 10^{-9}$ \\
\hline 0.5 & 0.677270 & 0.677270 & $4.974440 \times 10^{-9}$ \\
\hline 0.6 & 0.764037 & 0.764037 & $4.021380 \times 10^{-9}$ \\
\hline 0.7 & 0.838044 & 0.838044 & $2.454450 \times 10^{-10}$ \\
\hline 0.8 & 0.901001 & 0.901001 & $2.836300 \times 10^{-9}$ \\
\hline 0.9 & 0.954508 & 0.954508 & $4.201010 \times 10^{-9}$ \\
\hline 1.0 & 1.000000 & 1.000000 & $1.110220 \times 10^{-16}$ \\
\hline
\end{tabular}


Table 6. Comparisons of the HAM and numerical solution.

\begin{tabular}{cccc}
\hline$\phi(\eta)$ & HAM Solution & Numerical Solution & Absolute Error \\
\hline 0.0 & 0.000000 & $2.528420 \times 10^{-8}$ & $2.528420 \times 10^{-8}$ \\
\hline 0.1 & 0.025998 & 0.025998 & $2.009500 \times 10^{-8}$ \\
\hline 0.2 & 0.074274 & 0.074274 & $1.458960 \times 10^{-8}$ \\
\hline 0.3 & 0.144049 & 0.144049 & $5.628440 \times 10^{-8}$ \\
\hline 0.4 & 0.233238 & 0.233238 & $1.590860 \times 10^{-8}$ \\
\hline 0.5 & 0.338896 & 0.338896 & $1.758020 \times 10^{-8}$ \\
\hline 0.6 & 0.457662 & 0.457662 & $1.656760 \times 10^{-8}$ \\
\hline 0.7 & 0.586132 & 0.586132 & $8.921160 \times 10^{-9}$ \\
\hline 0.8 & 0.721129 & 0.721129 & $3.159840 \times 10^{-9}$ \\
\hline 0.9 & 0.589864 & 0.589864 & $1.324590 \times 10^{-9}$ \\
\hline 1.0 & 1.000000 & 1.000000 & $2.220450 \times 10^{-16}$ \\
\hline
\end{tabular}

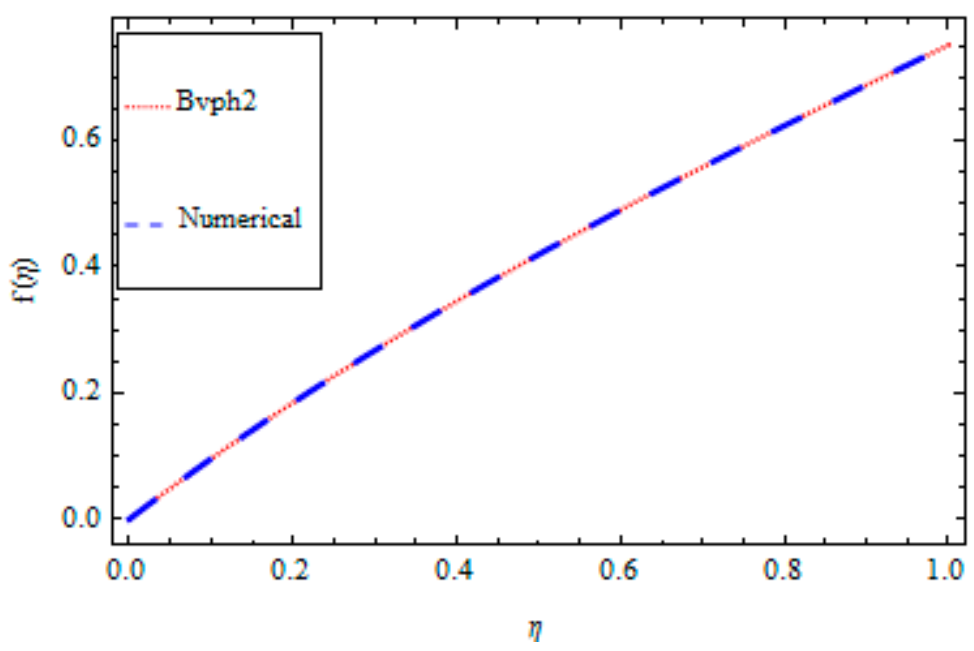

Figure 2. Graphical comparison of the HAM and numerical solution.

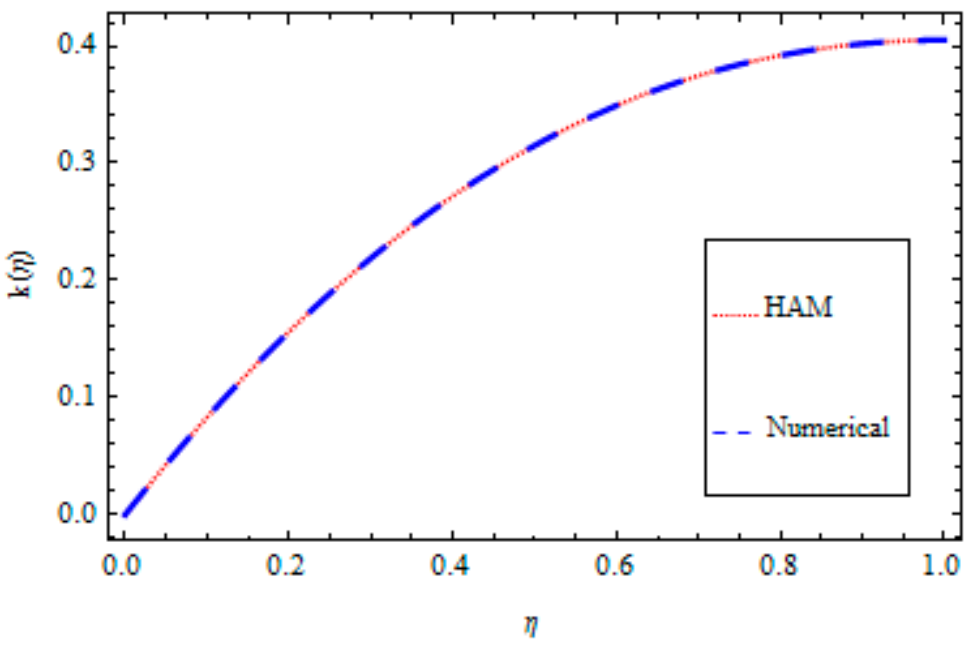

Figure 3. Graphical comparison of the HAM and numerical solution. 


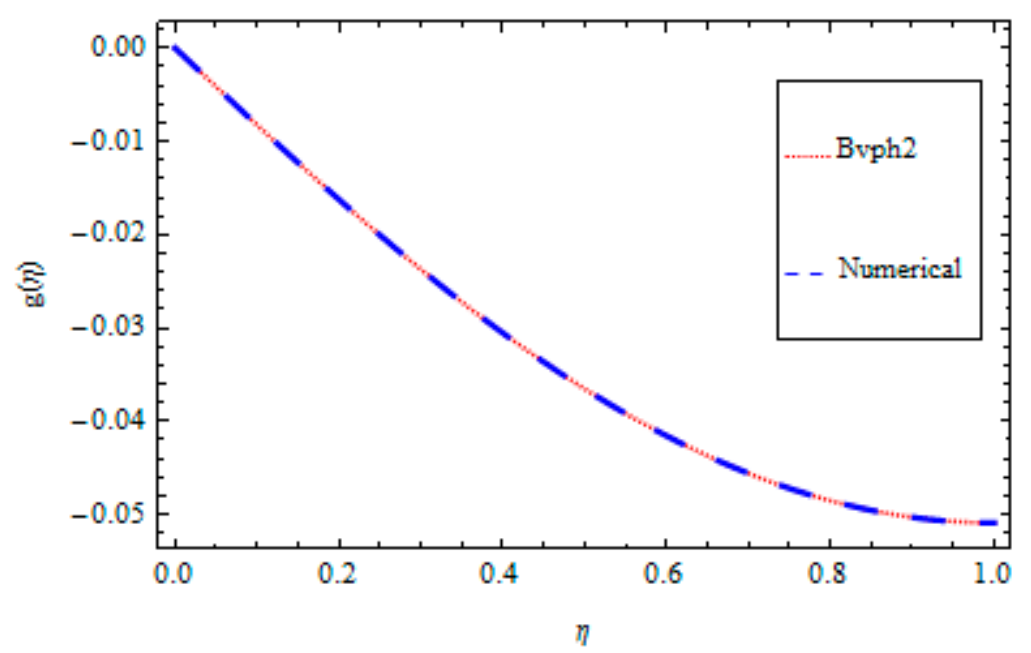

Figure 4. Graphical comparison of the HAM and numerical solution.

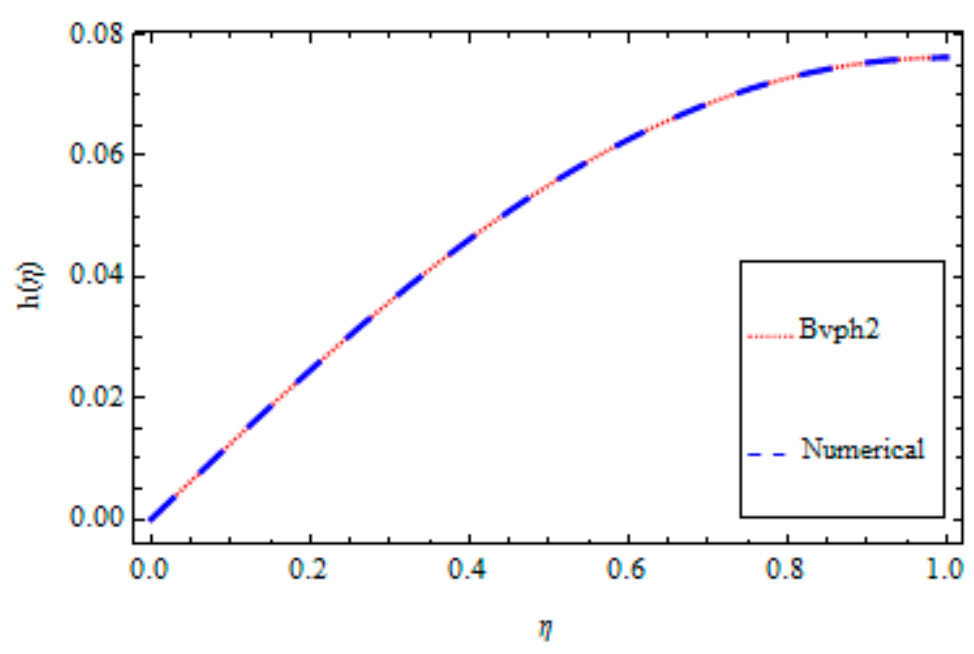

Figure 5. Graphical comparison of the HAM and numerical solution.

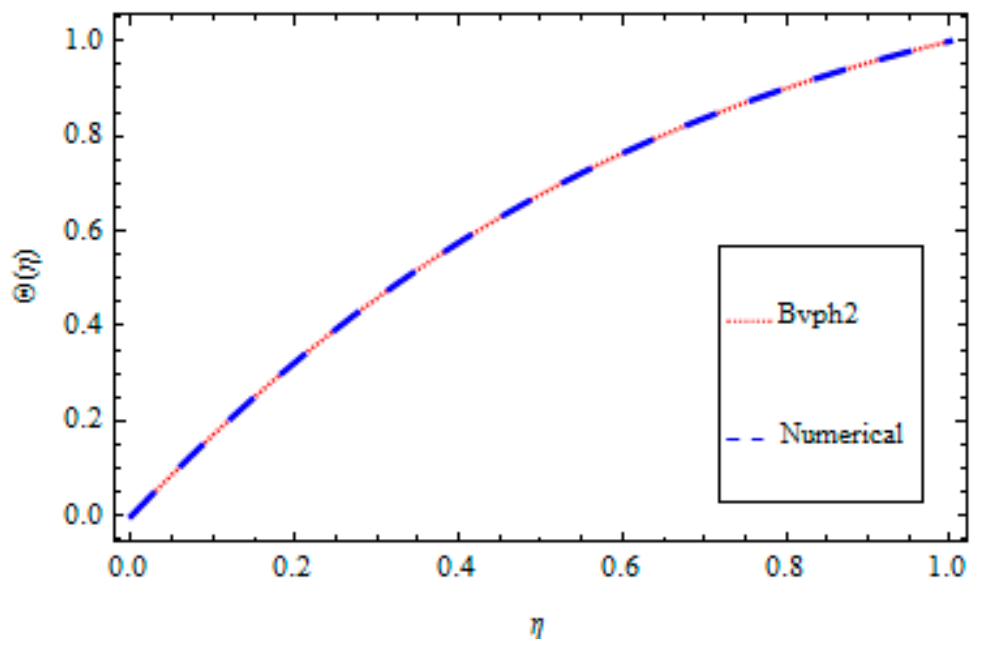

Figure 6. Graphical comparison of the HAM and numerical solution. 


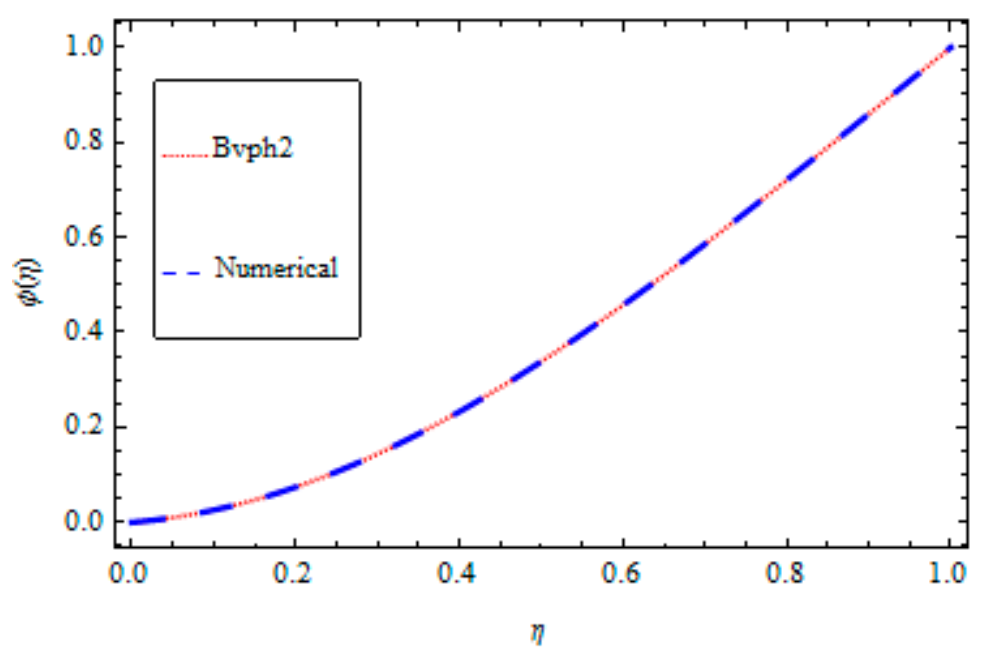

Figure 7. Graphical comparison of the HAM and numerical solution.

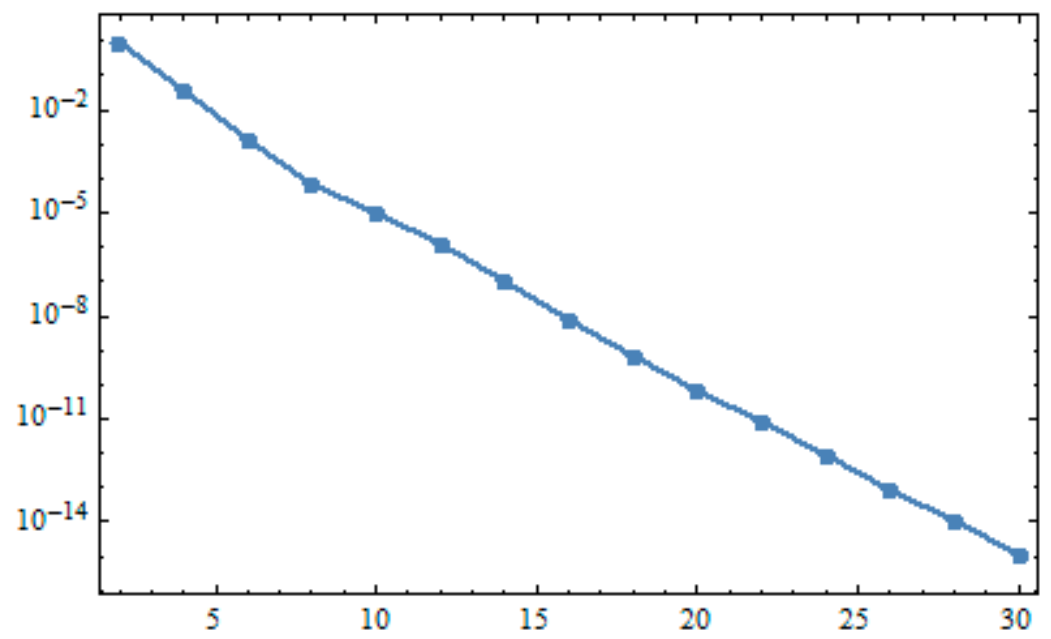

Figure 8. Error decay for the 10th-order approximation.

\section{Results and Discussion}

The three-dimensional thin film nanofluid flow for transfer of heat and mass across a spinning angled surface was observed. The analytical solution was obtained by the homotopy asymptotic method (HAM). For the accuracy of the results, the numerical BVPh2-midpoint method was also applied and good agreement was found. Additionally, the error decay for the 10th-order approximation was also calculated, which gave further validation to the method.

Figures 9-12 show how the unsteadiness factor $S$ affects the axial and radial velocity as well as the drainage and induced flow. The increase in $S$ increases the thickness of the momentum boundary layer. As a result, the previous mentioned form of the fluid motion decays. 


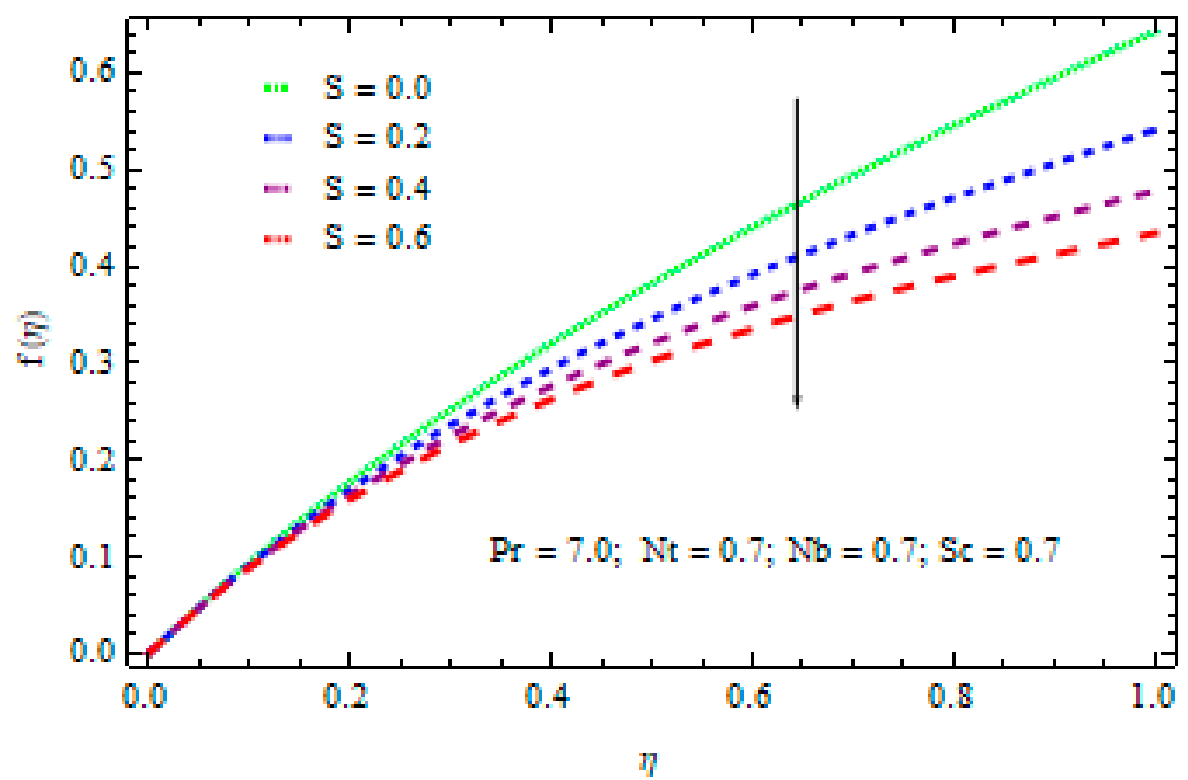

Figure 9. The impact of the unsteady parameter $S$ on the axial velocity.

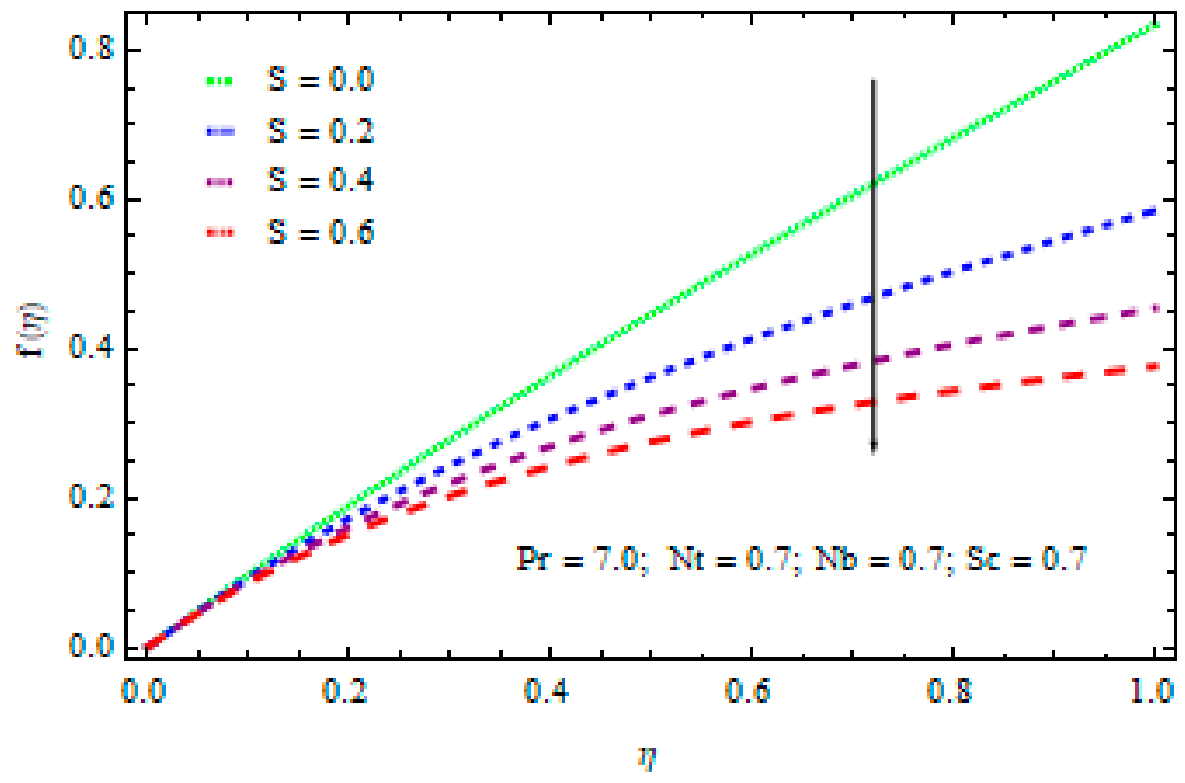

Figure 10. The impact of the unsteady parameter $S$ on the radial velocity. 


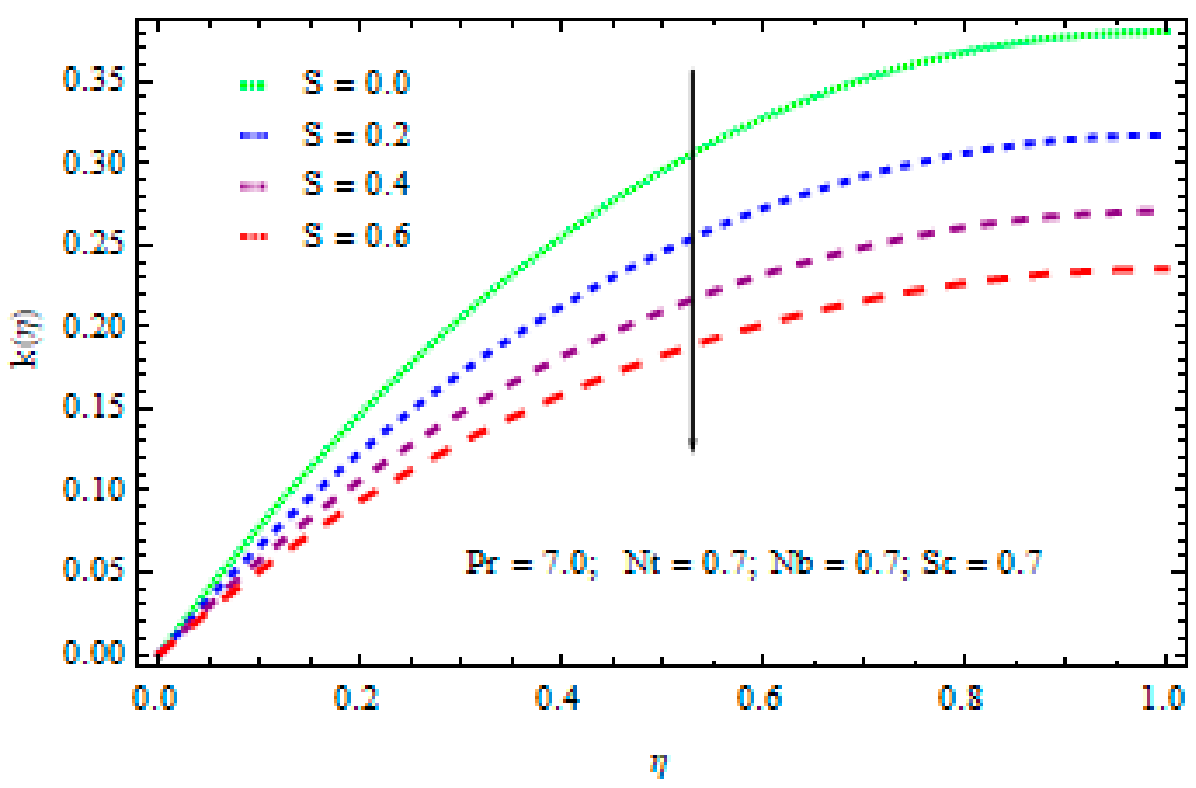

Figure 11. The impact of the unsteady parameter $S$ on the draining flow in the x-direction.

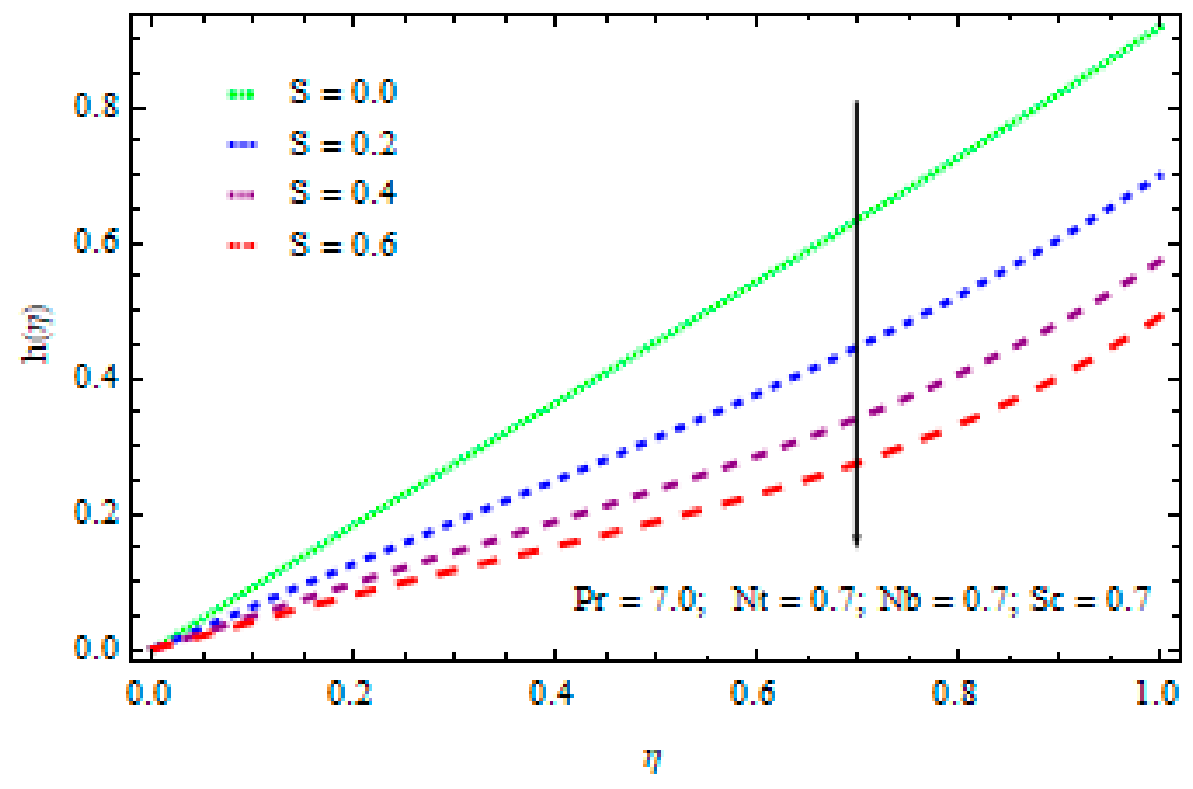

Figure 12. The impact of the unsteady parameter $S$ on the induced flow in the y-direction.

The temperature values drop with the rise in factor $S$ as depicted in Figure 13. The heat flow from sheet to fluid reduces with the rise in $S$ and results in a cooling effect. The collisions between fluid molecules are delayed to a small extent. Figure 14 shows a rise in the concentration profile. This is an impact of the rise in the momentum boundary layer resulting from the rise in the unsteadiness parameter $S$. 


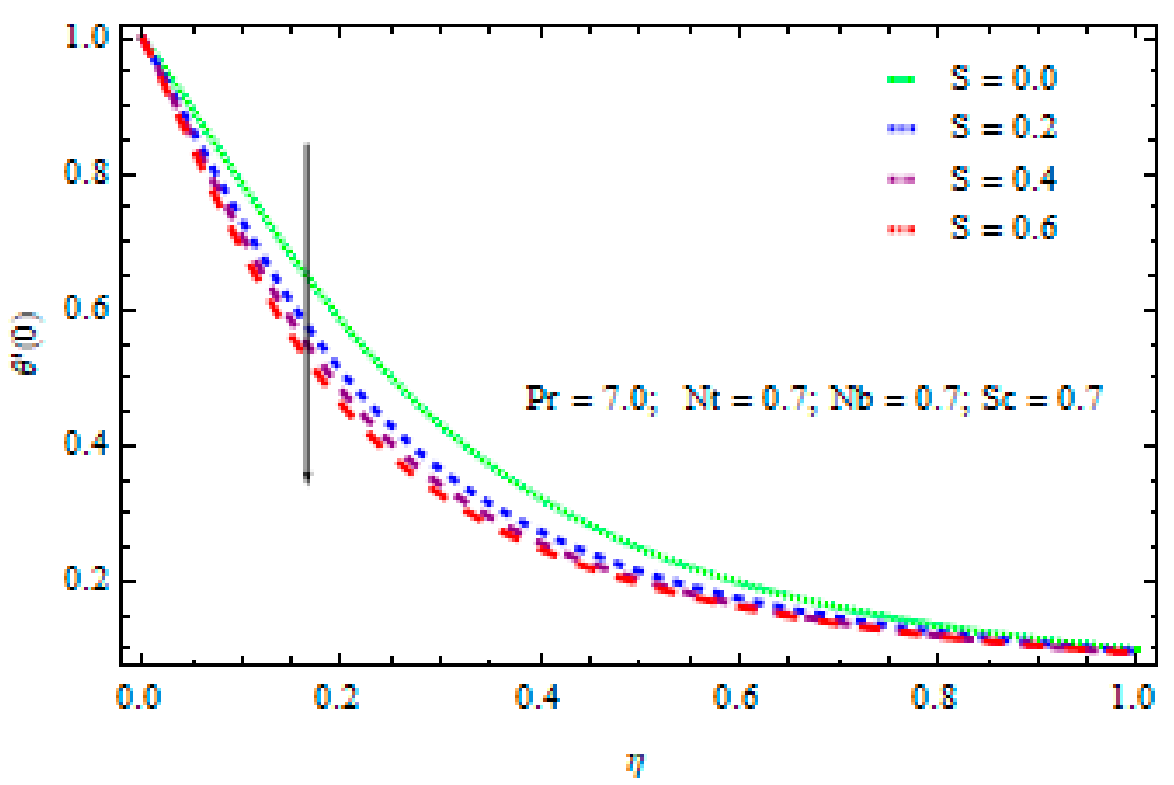

Figure 13. The impact of the unsteady parameter $S$ on the temperature field.

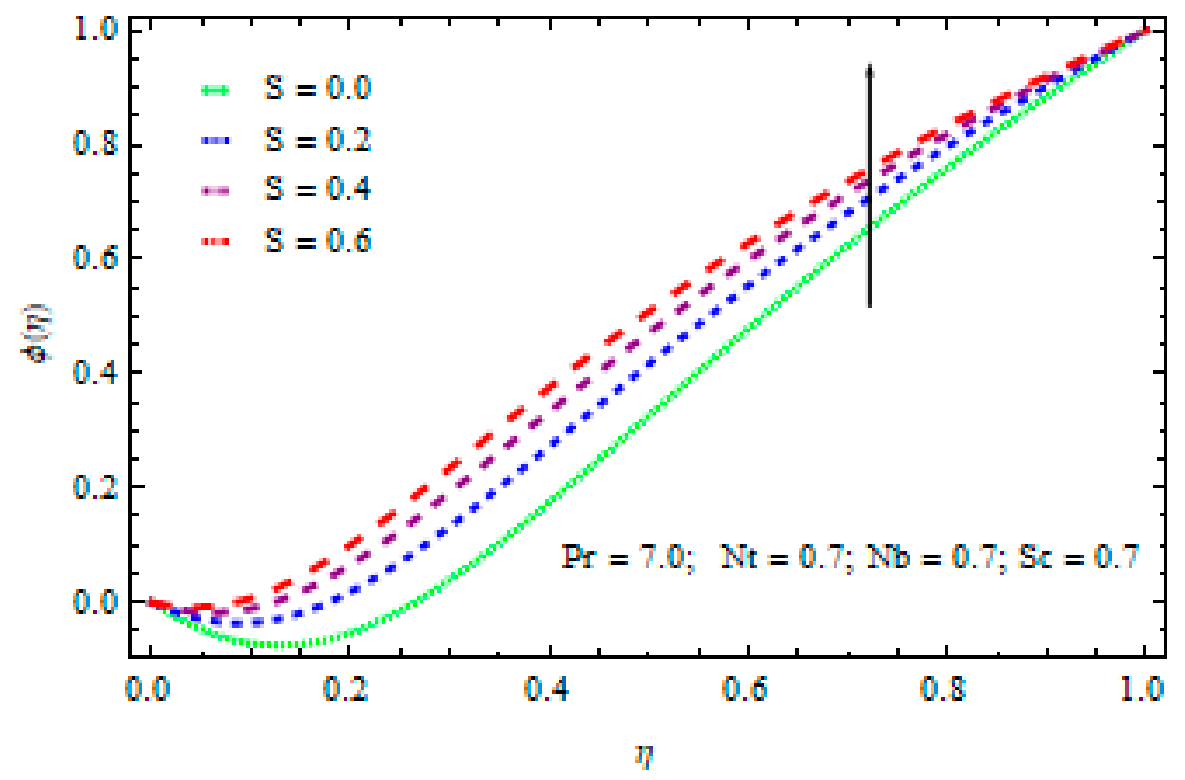

Figure 14. The impact of the unsteady parameter $S$ on the concentration.

Figure 15 shows how the Nusselt number varies with the unsteadiness parameter $S$. The rise in $S$ decreases the temperature of the momentum boundary layer, increasing the Nusselt number. This cooling effect is delayed because of the collisions of the molecules. Figure 16 shows a drop in the Sherwood number as the value of $S$ increases. Figure 17 shows the increase in the heat transfer as the value of $\mathrm{Nt}$ and $\mathrm{Nb}$ increase. The thickness of the thermal boundary layer increases with the increase in the Brownian motion represented by $\mathrm{Nb}$. 


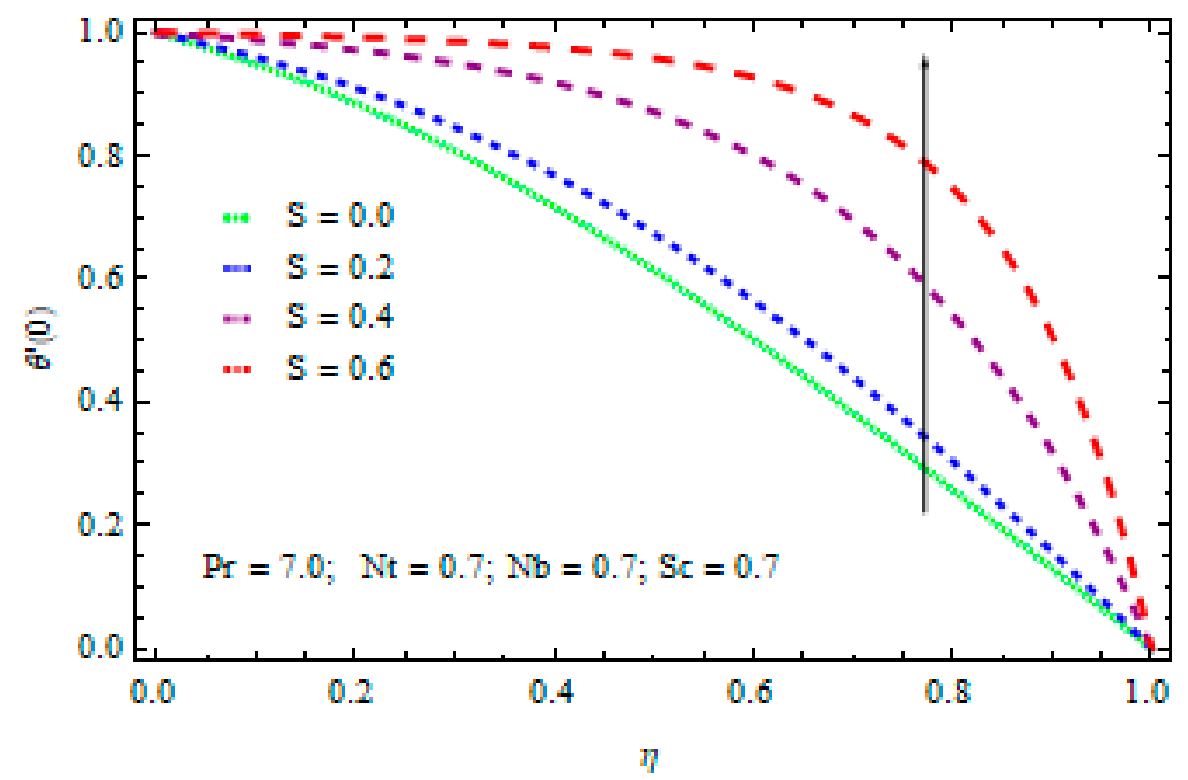

Figure 15. The impact of the unsteady parameter $S$ on the heat transfer rate.

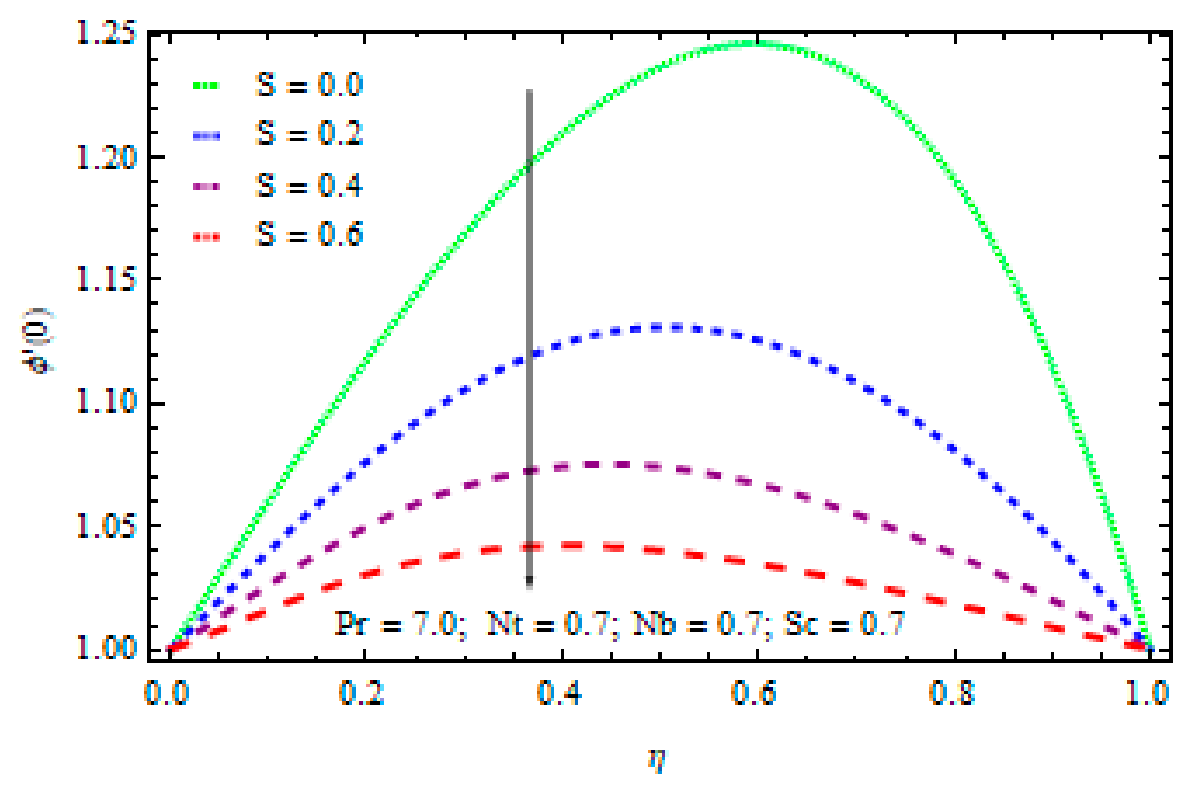

Figure 16. The impact of the unsteady parameter $S$ on the Sherwood number $S h$. 


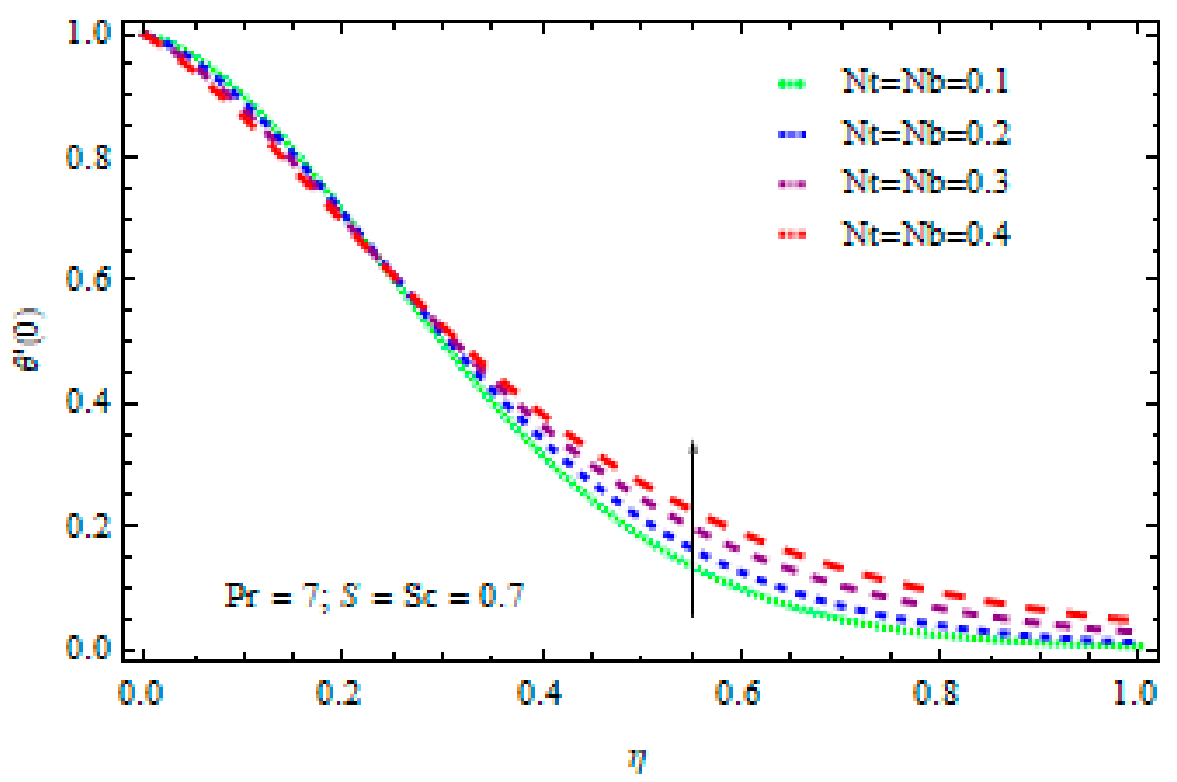

Figure 17. The impact of the $N t, N b$ parameters on the heat transfer rate.

Figure 18 represents the decline in the concentration rate with the variation of the Schmidt number Sc. Kinematic velocity is increased with the increase in the Schmidt number Sc, which reduces the Sherwood number because of the concentration of chemical species. Figure 19 shows the relationship of the Prandtl number $\mathrm{Pr}$ and the heat flow rate. Thickness of the thermal boundary increases with the increase in the Prandtl number, hindering the cooling process resulting from transfer of heat.

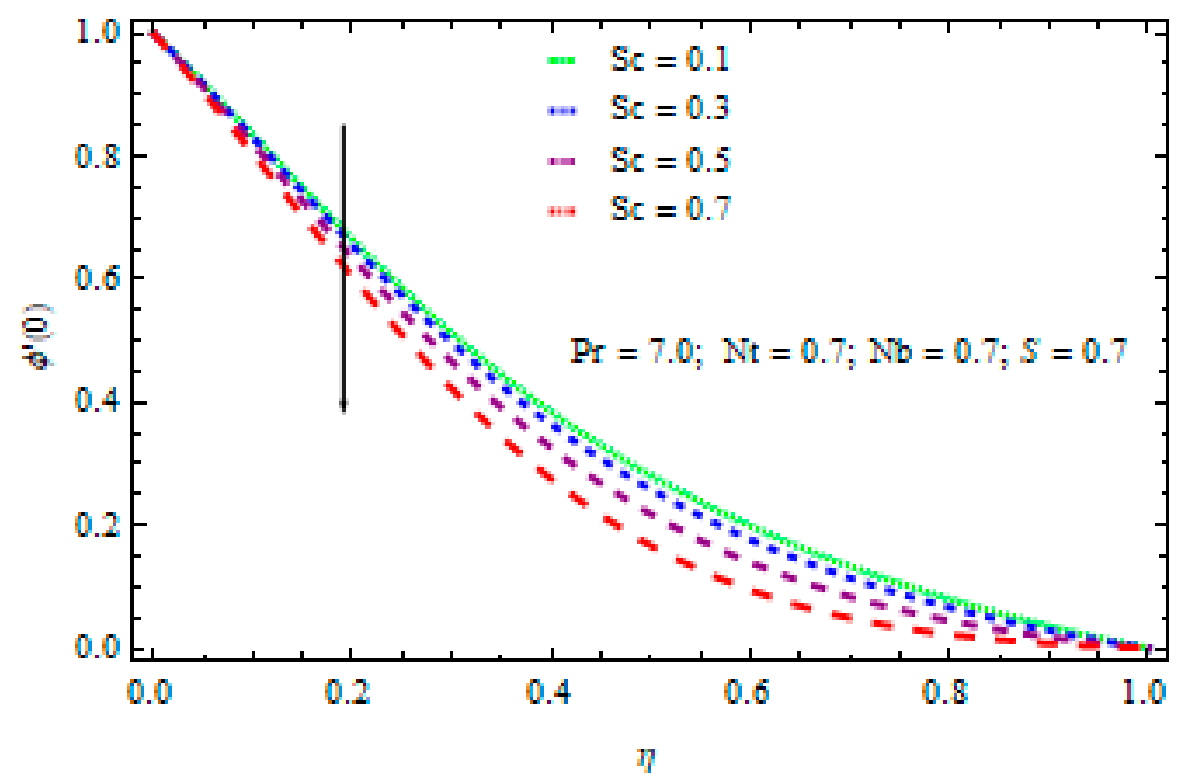

Figure 18. The impact of the $S c$ parameter on the Sherwood number. 


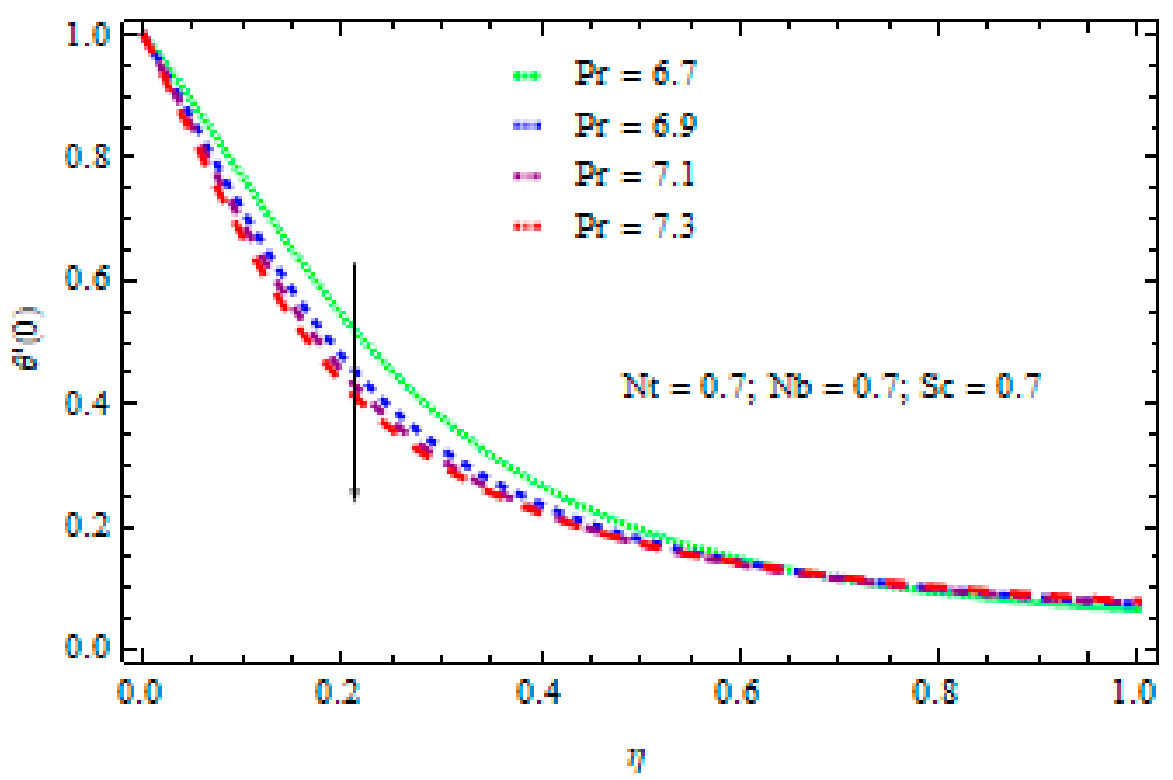

Figure 19. The impact of the $\operatorname{Pr}$ parameter on the heat transfer rate.

\section{Conclusions}

Published literature has mostly related to two-dimensional flow problems. Here, unsteady three-dimensional flow of a thin-film was investigated numerically over an inclined angular disk spayed with nanoparticles. The novelty of this study is that it is the first time HAM and BVPh2-midpont methods have been used to solve the modeled problem. The new results observed can be summarized as follows:

- The increase in unsteadiness factor $S$ increases the thickness of the momentum boundary layer.

- The temperature values drop with the rise in factor $\mathrm{S}$. The heat flow from sheet to fluid reduces with the rise in $S$ and results in a cooling effect.

- The impact of the rise in the momentum boundary layer resulted from the rise in the unsteadiness parameter $\mathrm{S}$.

- The rise in $\mathrm{S}$ decreases the temperature of the momentum boundary layer, increasing the Nusselt number. This cooling effect is delayed because of the collisions of the molecules.

- The Sherwood number drops as the value of $S$ increases.

- The thickness of the thermal boundary layer increases with the increase in the Brownian motion $\mathrm{Nb}$.

- Kinematic velocity is increased with the increase in the Schmidt number Sc. This reduces the Sherwood number because of the concentration of chemical species.

- Thickness of the thermal boundary layer increases with the increase in the Prandtl number Pr, hindering the cooling process resulting from transfer of heat.

Author Contributions: Investigation and Methodology: H.U.R.; Formal Analysis and Resources: Z.K.; Software and Supervision: I.K.; Validation and Writing and Review: D.L.C.C.; Funding Acquisition: K.S.N.

Acknowledgments: Authors would like to thanks YUTP 0153AB 078 for the financial support.

Conflicts of Interest: The authors declare that they have no conflict of interest.

\section{References}

1. Miladinova, S.; Lebon, G.; Toshev, E. Thin film flow of a power law liquid falling down an inclined plate. J. Non-Newton. Fluid Mech. 2004, 122, 69-78. [CrossRef]

2. Gul, T.; Shah, R.A.; Islam, S.; Arif, M. MHD thin film flows of a third-grade fluid on a vertical belt with slip boundary conditions. J. Appl. Math. 2013, 2013, 707286. [CrossRef] 
3. Khaled, A.R.; Vafai, K. Hydrodynamic squeezed flow and heat transfer over a sensor surface. Int. J. Eng. Sci. 2004, 42, 509-519. [CrossRef]

4. Siddiqui, A.M.; Ahmed, M.; Ghori, Q.K. Thin film flow of non-Newtonian fluid on a moving belt. Chaos Sol. Fract. 2007, 33, 1006-1016. [CrossRef]

5. Siddiqui, A.M.; Mahmood, R.; Ghori, Q.K. Homotopy perturbation method for thin film flow of a fourth-grade fluid down a vertical cylinder. Phys. Lett. A 2006, 352, 404-410. [CrossRef]

6. Costa, A.; Macedonio, G. Viscous heating in fluids with temperature dependent viscosity implications for magma flows. Nonlinear Proc. Geophys. 2003, 10, 545-555. [CrossRef]

7. Nadeem, S.; Awais, M. Thin film flow of an unsteady shrinking sheet through porous medium with variable viscosity. Phys. Lett. A 2008, 372, 4965-4972. [CrossRef]

8. Ellahi, R.; Riaz, A. Analytical solution for MHD flow in a third-grade fluid with variable viscosity. Math. Comput. Mod. 2010, 52, 1783-1793. [CrossRef]

9. Aksoy, Y.; Pakdemirly, M. Approximate analytical solution for flow of a third-grade fluid through a parallel-plate channel filled with a porous medium. Transp. Porous 2010, 83, 375-395. [CrossRef]

10. Sheikholeslami, M.; Hatami, M.; Ganji, D.D. Numerical investigation of nanofluid spraying on an inclined rotating disk for cooling process. J. Mol. Liq. 2015, 211, 577-583. [CrossRef]

11. Vajravelu, K.; Sreenadh, S.; Saravana, R. Influence of velocity slip and temperature jump conditions on the peristaltic flow of a Jeffrey fluid in contact with a Newtonian fluid. Appl. Math. Nonlinear Sci. 2017, 2, 429-442. [CrossRef]

12. Prasad, K.V.; Vaidya, H.; Vajravelu, K. MHD mixed convection heat transfer over a non-linear slender elastic sheet with variable fluid properties. Appl. Math. Nonlinear Sci. 2017, 2, 351-366. [CrossRef]

13. Awati, V.B. Dirichlet series and analytical solutions of MHD viscous flow with suction/blowing. Appl. Math. Nonlinear Sci. 2017, 2, 341-350. [CrossRef]

14. Attia, H.A. Unsteady MHD flow near a rotating porous disk with uniform suction or injection. Fluid Dyn. Res. 1998, 23, 283-290. [CrossRef]

15. Freidoonimehr, N.; Rashidi, M.M.; Mahmud, S. Unsteady MHD free convective flow past a permeable stretching vertical surface in a nano-fluid. Int. J. Therm. Sci. 2015, 87, 136-145. [CrossRef]

16. Makinde, O.D.; Mabood, F.; Khan, W.A.; Tshehla, M.S. MHD flow of a variable viscosity nanofluid over a radially stretching convective surface with radiative heat. J. Mol. Liq. 2016, 219, 624-630. [CrossRef]

17. Akbar, T.; Batool, S.; Nawaz, R.; Zia, Q.M.Z. Magnetohydrodynamics flow of nanofluid due to stretching/shrinking surface with slip effect. Adv. Mech. Eng. 2017, 9. [CrossRef]

18. Ramzan, M.; Chung, J.D.; Ullah, N. Partial slip effect in the flow of MHD micropolar nanofluid flow due to a rotating disk-A numerical approach. Results Phys. 2017, 7, 3557-3566. [CrossRef]

19. Hilfer, R. Applications of Fractional Calculus in Physics; World Scientific Publishing Company: Singapore, 2000; pp. 87-130.

20. Sabatelli, L.; Keating, S.; Dudley, J.; Richmond, P. Waiting time distributions in financial markets. Eur. Phys. J. B 2002, 27, 273-275. [CrossRef]

21. Metler, R.; Klafter, J. The random walk's guide to anomalous diffusion: A fractional dynamics approach. Phys. Rep. 2000, 339, 1-77. [CrossRef]

22. Khan, Z.; Rasheed, H.; Tlili, I.; Khan, I.; Abbas, T. Runge-Kutta 4th-order method analysis for viscoelastic Oldroyd 8-constant fluid used as coating material for wire with temperature dependent viscosity. Sci. Rep. 2018. [CrossRef] [PubMed]

23. Khan, Z.; Rasheed, H.; Ullah, M.; Gul, T.; Jan, A. Analytical and numerical solutions of Oldroyd 8-constant fluid in double-layer optical fiber coating. J. Coat. Technol. Res. 2019, 16, 235-248. [CrossRef]

24. Khan, M.; Abbas, Q.; Duru, K. Magnetohydrodynamic flow of a Sisko fluid in annular pipe: A numerical study. Int. J. Numer. Meth. Fluids 2010, 62, 1169-1180. [CrossRef]

25. Saeed, A.; Shah, Z.; Islam, S.; Jawad, M.; Ullah, A.; Gul, T.; Kumam, P. Three-Dimensional Casson Nanofluid Thin Film Flow over an Inclined Rotating Disk with the Impact of Heat Generation/Consumption and Thermal Radiation. Coatings 2019, 9, 248. [CrossRef]

26. Binding, D.M.; Blythe, A.R.; Guster, S.; Mosquera, A.A.; Townsend, P.; Wester, M.P. Modelling polymer melt flows in wire coating process. J. Non-Newton. Fluid Mech. 1996, 64, 191-206. [CrossRef]

27. Nayak, M.; Dash, G.C.; Singh, L.P. Steady MHD flow and heat transfer of a third grade fluid in a wire coating analysis with temperature dependent viscosity. Int. J. Heat Mass Transf. 2014, 79, 1087-1095. [CrossRef] 
28. Salem, A.M. Variable viscous and thermal conductivity effect on MHD flow and heat transfer in viscoelastic fluid over a stretching sheet. Phys. Lett. A 2007, 369, 315-322. [CrossRef]

29. Bhukta, D.; Dash, G.C.; Mishra, S.R.; Baag, S. Dissipation effect on MHD mixed convection flow over a stretching sheet through porous medium with non-uniform heat source/sink. Ain Shams Eng. J. 2015, 8, 353-361. [CrossRef]

30. Majeed, A.; Zeeshan, A.; Ellahi, R. Unsteady ferromagnetic liquid flow and heat transfer analysis over a stretching sheet with the effect of dipole and prescribed heat flux. J. Mol. Liq. 2016, 223, 528-533. [CrossRef]

31. Liao, S.J. Beyond Perturbation: Introduction to Homotopy Analysis Method; Chapman and Hall, CRC Press: Boca Raton, FL, USA, 2003.

32. Liao, S.J. Homotopy Analysis Method in Non-Linear Differential Equations; Springer and Higher Education Press: Heidelberg, Germany, 2012.

33. Khan, Z.; Khan, M.A.; Siddiqui, N.; Ullah, M.; Shah, Q. Solution of magnetohydrodynamic flow and heat transfer of radiative viscoelastic fluid with temperature dependent viscosity in wire coating analysis. PLoS ONE 2018, 13, e0194196. [CrossRef] [PubMed]

34. Khan, Z.; Rasheed, H.; Alkanhal, T.A.; Ullah, M.; Khan, I.; Tlili, I. Effect of magnetic field and heat source on Upper-convected-maxwell fluid in a porous channel. Open Phys. 2018, 16, 917-928. [CrossRef]

(C) 2019 by the authors. Licensee MDPI, Basel, Switzerland. This article is an open access article distributed under the terms and conditions of the Creative Commons Attribution (CC BY) license (http://creativecommons.org/licenses/by/4.0/). 